Electronic Journal of Research in Educational Psychology

- (1) ejrep
Electronic Journal of Research in Educational Psychology

E-ISSN: 1696-2095

jfuente@ual.es

Universidad de Almería

España

Ramírez Dorantes, María del Carmen; Canto y Rodríguez, José Enrique Desarrollo y evaluación de una escala de autoeficacia en la elección de carrera en estudiantes mexicanos

Electronic Journal of Research in Educational Psychology, vol. 5, núm. 1, abril, 2007, pp. 37-56

Universidad de Almería

Almeria, España

Disponible en: http://www.redalyc.org/articulo.oa?id=293121941004

- Cómo citar el artículo

- Número completo

- Más información del artículo

- Página de la revista en redalyc.org

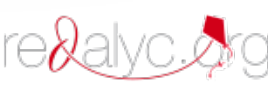

Sistema de Información Científica

Red de Revistas Científicas de América Latina, el Caribe, España y Portugal Proyecto académico sin fines de lucro, desarrollado bajo la iniciativa de acceso abierto 


\section{Desarrollo y evaluación de una escala de auto- eficacia en la elección de carrera en estudiantes mexicanos}

\section{María del Carmen Ramírez Dorantes ${ }^{1}$, José Enrique Canto y Rodríguez ${ }^{2}$}

${ }^{1}$ Dirección General de Desarrollo Académico, Universidad Autónoma de Yucatán, ${ }^{2}$ Facultad de Psicología, Universidad Autónoma de Yucatán

México

rdorant@uady.mx

crodri@uady.mx 


\section{Resumen}

Introducción: En los últimos años, los investigadores iniciaron el estudio del papel que juegan las creencias de autoeficacia para la comprensión de los procesos para la elección de carrera, así como el estudio acerca de la efectividad de la exploración de las opciones de ocupación y decidir por una carrera (Hackett y Betz, 1995). El propósito de esta investigación fue desarrollar y evaluar una escala de autoeficacia para la elección de carrera con tareas culturalmente significativas para estudiantes de bachillerato de México.

Método: La muestra estuvo compuesta por 343 estudiantes de dos escuelas de bachillerato. Se diseñó un instrumento a partir de la versión en español del Career Decisión-Making Selfefficacy Scale (Taylor y Betz, 1983). La versión final del instrumento quedó conformada por 55 items distribuidos en cinco subescalas: Autoconocimiento, Información ocupacional, Selección de metas, Solución de problemas, y Planeación.

Resultados: El instrumento desarrollado mostró estar constituido por cinco factores que explican el $42.2 \%$ de la varianza total, distribuidos del factor 1 (Autoconocimiento) al 5 (Planeación) de la siguiente manera: 30.4\%, 3.9\%, 2.7\%, 2.8\%, 2.4, respectivamente. Se observó claramente que es el primer factor (Autoconocimiento) el que explica la varianza, principalmente. Además, se encontró que el instrumento hallado tiene una fiabilidad total alta (alfa de Cronbach) igual a 0.95, y las fiabilidades de los factores fluctuaron de 0.79 (Autoconocimiento) a 0.88 (Planeación).

Discusión: La inclusión de tareas culturalmente apropiadas a los estudiantes de preparatoria redundó en una mejor reproducción de la estructura teórica de la autoeficacia en la elección de carrera. El enfoque teórico estableció la existencia de cinco dimensiones que la definen, y dichas dimensiones fueron recreadas mediante la combinación de items originales e items nuevos. Sin embargo, es importante observar que el factor Autoconocimiento tiene mucho peso para explicar la autoeficacia para la elección de carrera. El instrumento mostró tener una confiabilidad robusta.

Palabras Clave: Autoeficacia, autoeficacia en la elección de carrera, medición, bachillerato. 


\section{Introducción}

La congruencia entre la persona y su profesión contribuye significativamente a la satisfacción con la vida y la autoestima personal (McIntosh, 2000). De esta manera, se puede afirmar que la elección de carrera es una de las principales tareas que tienen que realizar las personas, ya que una adecuada elección puede significar un mejor desarrollo y una estabilidad emocional adecuada. Debido a la enorme cantidad de opciones educativas y profesionales a las que tienen acceso los estudiantes en la actualidad, los orientadores educativos están cada vez más preocupados para colaborar con ellos en la realización de una adecuada elección de carrera.

Elegir una carrera es una de las tareas más críticas e importantes a las que se enfrentan los estudiantes, ya que determina en gran parte la manera en que invertirán su tiempo, quiénes serán sus amigos, cuáles serán los valores y las actitudes que adoptarán en el futuro, así como el lugar en donde vivirán, y cómo podría ser el patrón de su vida familiar (Hackett y Betz, 1995).

La situación anterior ha llamado la atención de los investigadores de los fenómenos vocacionales, quienes se han dado a la tarea de estudiar cómo se realiza el proceso de elección de carrera. Para ello han hecho uso de uno de los constructos más recientes empleados para explicar dicho proceso, como es el de las creencias de autoeficacia (Bandura, 1977, 1986, 1997). El constructo de autoeficacia se refiere a las creencias individuales acerca de las propias habilidades para llevar a cabo las actividades requeridas para producir determinados resultados. Estas creencias juegan un papel fundamental en el control de la elección de la conducta, la persistencia, los estados emocionales y los procesos de pensamiento (Bandura, 1997).

Betz y Hackett (1981) emplean el concepto de autoeficacia en la elección de carrera como una extensión del constructo antes mencionado, y lo definen como las creencias que tienen las personas con respecto a su capacidad para elegir con éxito una carrera. Bandura (1997) afirma que "existe un cuerpo sustancial de investigación que muestra que las creencias de autoeficacia desempeñan un papel crucial en el desarrollo de las carreras y su búsqueda" (p. 423). 
Uno de los instrumentos de evaluación de carrera más comúnmente empleados es el Career Decision Making Self-Efficacy Scale (CDMSE) (Taylor y Betz, 1983). El CDMSE esta basado en la teoría social cognitiva de la autoeficacia (Bandura, 1977, 1986, 1997; Taylor y Betz, 1983). La teoría de la autoeficacia propuesta por Bandura es una de las teorías más recientes de una larga tradición de teorías acerca de la competencia personal y la eficacia, y ha generado más investigaciones en psicología clínica, social y de la personalidad en las últimas dos décadas y media que otros modelos y teorías relacionadas (Maddux, 1995).

Hackett y Betz (1981) ampliaron la teoría de la autoeficacia al aplicarla al área de la autoeficacia vocacional, que se asocia con las tareas relacionadas para elegir carrera. Esta nueva área fue dividida a su vez en dos áreas: una que estudia el contenido de la elección, y otra que se refiere a los procesos que las personas emplean para realizar su elección de carrera. Mediante la primera área se estudia el tipo de carrera específico que se elige, ya sea matemáticas, literatura o ciencias. Mientras que la segunda se dirige a estudiar cuáles son los procesos (conductas) que las personas realizan para elegir una carrera.

Algunos estudios han mostrado relaciones significativas entre las puntuaciones de la CDMSE y la actividad de exploración para encontrar carreras (Blustein, 1989), las habilidades y las actitudes (Luzzo, 1996), la indecisión vocacional (Robbins, 1985, Taylor y Betz, 1983, Taylor y Popma, 1990), y la identidad vocacional y la autoestima (Robbins, 1985).

Basándose en la teoría de autoeficacia de Bandura y en la distinción de Hackett y Betz entre el contenido de la elección de carrera y los procesos de elección vocacional, tanto como en las nociones de Crites (1978) acerca de las competencias de la elección de carrera, Taylor y Betz, (1983), desarrollaron el CDMSE. Crites (1978) propuso cinco procesos de elección de carrera que resultarían en decisiones vocacionales óptimas: Autoconocimiento (SelfAppraisal), Información ocupacional (Gathering occupational information), Establecimiento de metas (Goal selection), Planeación (Making plans for the future), Solución de problemas (Problem solving).

El CDMSE mide el grado en que los individuos tienen confianza en su habilidad para completar tareas relacionadas para la toma de decisiones vocacionales. Algunas versiones cortas del CDMSE han sido desarrolladas y modificadas para ciertos grupos de estudiantes 
como los de educación secundaria y los de bachillerato (Anderson y Brown, 1997; Betz, Klein y Taylor, 1996; Carns et al, 1995).

En México, Canto y Rodríguez (1998) traducen al español la versión reducida del CDMSE (Career Decision Making Self-Efficacy Scale-Brief Form) (Betz, Klein y Taylor, 1996) y lo administró a estudiantes del último curso de Bachillerato. Este instrumento contiene las mismas cinco subescalas y consta de 25 items. Los resultados del análisis factorial indicaron inconsistencias tanto en el número de dimensiones recreadas como en la agrupación de los items en las subescalas propuestas en el instrumento original. Basándose en los hallazgos de Pajares (1996), se estableció una hipótesis para explicar los resultados obtenidos por Canto y Rodríguez (1998). Pajares, estudiando la teoría de la autoeficacia en contextos hispanoamericanos, encontró que las relaciones con otros constructos dependen de lo significativo que resulten las tareas a los estudiantes. De acuerdo con esto, probablemente, las tareas contenidas en el instrumento utilizado, carecían de signicatividad para los estudiantes latinos. Pajares, sin embargo, no especifica el proceso que se activa cuando el estudiante responde a items con tareas poco familiares.

Debido a los resultados anteriores y a la escasez de instrumentos válidos y confiables que sirvan para diagnosticar y apoyar, en su caso, el proceso de elección de carrera de los estudiantes mexicanos, se planteó como propósito de este estudio desarrollar y evaluar una escala de autoeficacia para la elección de carrera que contuviera tareas culturalmente apropiadas para estudiantes de bachillerato de México.

\section{Método}

\section{Participantes}

La población del estudio estuvo constituida por todos los estudiantes matriculados en los tres cursos de las escuelas de bachillerato Uno y Dos de la Universidad Autónoma de Yucatán (UADY).

De acuerdo con los datos proporcionados por el Departamento de Servicios Escolares de la propia Universidad, en el momento del estudio se encontraban matriculados 5705 estudiantes en las dos preparatorias de la UADY, distribuidos de la siguiente manera: en la Preparatoria Uno, 3210 estudiantes; 1 279, en primer curso; 923, en segundo, y 1008 en tercero. 
En la Preparatoria Dos se inscribieron 2 495, distribuidos de la siguiente manera: 898 en primer curso, 804 en segundo y 793 en tercero.

Se seleccionó una muestra aleatoria estratificada proporcional, en la que los estratos se formaron por los tres grados escolares, para asegurar la representatividad de los tres cursos escolares en la muestra.

La muestra seleccionada estuvo compuesta por 343 estudiantes, con una edad promedio de 18.5 años y un promedio de rendimiento académico de 79. De dicha muestra, 188 estudiantes $(54.8 \%)$ estaban inscritos a la preparatoria Uno y $155(45.2 \%)$ a la Preparatoria Dos; 178 (51.9\%) fueron mujeres y 165 (48.1\%) hombres; 148 (43.1\%) estaban matriculados a primer curso, $82(23.9 \%)$ a segundo y $113(32.9 \%)$ a tercero. Los criterios para seleccionar a estas dos preparatorias de la UADY fueron el conocimiento de las características de las poblaciones y que se considera que son una muestra representativa de los estudiantes de bachillerato de las demás preparatorias existentes en la ciudad. Además, se consideró el número de estudiantes inscritos a cada una de las preparatorias y el número de estudiantes que se requiere para cubrir el objetivo del estudio.

\section{Instrumento}

La Escala de Autoeficacia para la Elección de Carrera (EAEC) (Anexo 1) que fue la versión final del instrumento, mantuvo la estructura teórica establecida para la autoeficacia en la elección de carrera. Su objetivo fue medir la confianza que sienten los estudiantes para realizar con éxito cada una de las tareas requeridas en su proceso de elección vocacional. $\mathrm{Su}$ desarrollo se basó en la versión traducida del CDMSE (Taylor y Betz, 1983). La longitud del instrumento estuvo dada por 55 items de los cuales 28 provenían del CDMSE por considerar, de acuerdo con una evaluación de jueces, que el contenido representaba un significado adecuado para la realidad de los estudiantes mexicanos y los restantes 27 items, fueron desarrollados por el primer autor. Los items quedaron distribuidos en las mismas cinco subescalas del CDMSE: Autoconocimiento, 11 items; Información ocupacional, 10; Selección de metas, 13; Solución de problemas, 12 y Planeación 9.

Para responder a la escala, se le pidió al estudiante que en una escala de 10 puntos, que va de ninguna confianza (0) a confianza absoluta (9), ubicara su respuesta de acuerdo con la 
confianza que siente poseer para realizar con éxito cada una de las tareas que se le presentan. Puntuaciones altas significaban niveles altos de autoeficacia en la elección de carrera.

\section{Procedimiento}

En una primera fase del estudio, se desarrolló una versión preliminar de la EAEC que contenía 63 items, de los cuales 36 provenían de la versión traducida al español del CDMSE y 27 items nuevos, que fueron diseñados con el fin de aumentar el número de items culturalmente significativos para la población del estudio. Finalmente los 63 items quedaron distribuidos de la siguiente manera: 13 de Autoconocimiento, 11 de Información ocupacional, 15 de selección de metas, 13 de Solución de problemas y 11 de Planeación.

La decisión de incorporar los 36 items del CDMSE estuvo guiada por el análisis del contenido de los items. El criterio que se siguió para el análisis fue la significancia de la tarea implícita en el item. Las tareas con escasa significancia fueron rechazadas. Por ejemplo, la tarea que implica el uso de bibliotecas para obtener información de las tendencias de empleo fue rechazada debido a su escasa significancia. Los items nuevos fueron diseñados para indagar tareas significativas particularmente para las áreas de establecimiento de metas y planeación.

Tres expertos en Orientación y Consejo en ejercicio con más de 10 años de experiencia en el área, evaluaron el contenido de los 27 items nuevos. El criterio para conservar el item fue que dos de los expertos coincidieran en la pertenencia del item a la subescala. De esta forma los 27 items nuevos fueron ubicados en las subescalas respectivas.

La versión preliminar se probó experimentalmente en una muestra de 200 estudiantes de preparatoria. Mediante el análisis de los índices de confiabilidad y de validez de los items se decidió eliminar 8 items todos ellos provenientes de los 36 items tomados del CDMSE. Los items eliminados provenían de las siguientes subescalas: dos de Autoconocimiento, uno de Información ocupacional, dos de Selección de metas, uno de Solución de problemas, y dos de Planeación.

La versión final del instrumento se administró a la muestra de 343 estudiantes de preparatoria. La estrategia general que se siguió para explorar la estructura factorial de la escala fue, primero, obtener la estructura factorial no rotada de los factores. La matriz de correlacio- 
nes de Pearson de los items fue analizada factorialmente usando el método de máxima verosimilitud (MMV) bajo la hipótesis de cinco factores no correlacionados. Segundo, se rotó la estructura factorial para tratar de mejorar la interpretación del significado de cada uno de los cinco factores. Se probaron los métodos varimax y quartimax.

La fiabilidad de la información derivada de la versión final fue investigada con el índice de consistencia interna Alfa de Cronbach para la escala total y cada una de las cinco subescalas.

\section{Análisis Estadístico}

Primero se realizó un análisis descriptivo de las variables demográficas de edad, sexo, escuela de bachillerato, curso y promedio de calificaciones para obtener la caracterización de la muestra.

Posteriormente, se llevó a cabo un análisis factorial de la estructura de la escala total, una estimación de los errores asociados a las cargas factoriales, un cálculo de la correlación de cada item con el total de la subescala a la que pertenece el item, sin incluirlo. También se realizó una estimación del índice de confiabilidad para cada subescala, y para la escala total.

\section{Resultados}

Previo al análisis factorial de los reactivos de la escala total, éstos fueron analizados mediante el test KMO y el test de esfericidad de Bartlett para conocer su idoneidad para realizar dicho análisis. Se obtuvo un KMO de .896, y el test de Bartlett fue de 2964.66, con una significancia igual a .000 , lo que confirmó que los reactivos podrían ser analizados mediante un análisis factorial. La matriz de correlaciones de Pearson de la estructura factorial entre los 55 items fue estimada a partir de las respuestas de los 343 participantes. El rango de correlaciones varió de .14 a .67 y se observó que una proporción significativa de las correlaciones se encontraban distribuidas entre .32 y .56. Los cinco factores hallados explicaron el $42.2 \%$ de la varianza total, distribuyéndose dicho porcentaje, del factor uno al cinco, de la siguiente manera: 30.4, 3.9, 2.7, 2.8, 2.4, respectivamente. Resulta claro que fue el factor 1 el que en su conjunto explicó la mayor cantidad de la varianza obtenida entre los cinco factores. 
Para realizar el análisis de las cargas factoriales de los items se consideró que sólo se retendrían aquellos items que tuvieran una carga factorial igual o mayor a \pm 0.40 , es decir, éste sería el criterio para asignar pertenencia del item a un factor (Bollen, 1989). Con base en el criterio anterior, 49 de los 55 items tuvieron cargas factoriales significativas. Los items 3 , 6, y 7, que originalmente pertenecen a la subescala de Autoconocimiento, los items 30 y 31 que pertenecen a la subescala de Selección de metas y el item 46 que pertenece a la subescala de Solución de problemas resultaron no significativos.

Para investigar sí la estructura hipotetizada podría ser reproducida con mayor precisión y poder ser interpretada mejor, los factores se redefinieron en una segunda fase del análisis factorial mediante una rotación factorial. Cuando la matriz de correlaciones fue rotada por medio de los métodos Varimax y Quartimax, el primer método fue el que reprodujo de una manera más homogénea la estructura hipotetizada, dando lugar a factores más interpretables. (Anexo 2).

El procedimiento para interpretar los factores obtenidos de la matriz rotada mediante el método Varimax consiste en analizar las variables o items con cargas factoriales iguales o mayores que \pm 0.40 y los contenidos de los items del cuestionario asociadas con estas variables. (Bollen, 1989). Como resultado de los criterios anteriores de interpretación, se obtuvo la siguiente descripción de los factores. Es importante mencionar, que al realizar la interpretación de los factores obtenidos mediante la rotación de la matriz de correlaciones se conservaron los nombres de las subescalas originales.

El Factor 1, Establecimiento de metas, mide el grado de creencias que poseen los estudiantes respecto a su competencia para determinar sus metas académicas y laborales. Quedó integrado por 9 items de Selección de metas y 2 items de Autoconocimiento. Los primeros nueve items $(22,23,24,25,26,27,28,29$ y 34$)$ representan la posibilidad de determinar tanto las metas laborales como académicas de los estudiantes, aunque algunas circunstancias no sean favorables. Los últimos dos items ( 2 y 4 ) reflejan la posibilidad de determinar un estilo de vida que involucra un trabajo ideal. De los 11 items, 4 (items 26, 27, 28 y 29) fueron desarrollados como nuevos y los siete restantes fueron tomados de la versión original en inglés de la escala. 
El Factor 2, Solución de problemas, mide el grado de creencias que tienen los estudiantes respecto a su competencia para encontrar estrategias o medios para solucionar problemas. Este factor sólo incluyó un agrupamiento de items (35, 36, 37, 38, 39, 40, 41, 42, 43, 44 y 45) que originalmente pertenecían a la subescala de Solución de problemas. De los 11 items que cargaron en el Factor 2, cuatro fueron items nuevos, (39, 40, 41 y 42) desarrollados especialmente para ser incluidos en la subescala, los restantes fueron tomados de la escala original del instrumento.

El Factor 3, Información ocupacional, se definió como el grado de creencias que tienen los estudiantes acerca de su capacidad para obtener información ocupacional. Todos los items que se agruparon en este factor pertenecían originalmente a la subescala de Información ocupacional. Los nueve items $(12,13,14,15,16,17,18,19$ y 21) que se agruparon en el Factor 3 tienen que ver con los diferentes medios a través de los cuales un estudiante puede obtener información acerca de las carreras u ocupaciones, como por ejemplo asistir a pláticas, revisar libros, preguntarle a algún maestro, entre otros.

El Factor 4, Planeación, define el grado de creencias que poseen los estudiantes acerca de su capacidad para planear sus actividades académicas. Esta etiqueta fue usada ya que solo una agrupación de variables que originalmente pertenecían a la subescala de Planeación cargaron en este factor. Mediante el análisis del contenido de los items se pudo determinar que todos los items se referían a actividades o estrategias de planeación como componentes para la determinación de metas académicas y/o ocupacionales. En total, nueve items se agruparon en este factor $(47,48,49,50,51,52,53,54$, y 55). De todos los agrupamientos que se formaron, el correspondiente al factor 4 fue el que mayor número de items nuevos agrupó, ya que fueron 6 los items nuevos $(49,50,51,53,54$ y 55) que cargaron en este factor.

Por último el Factor 5, Autoconocimiento, mide el grado de las creencias de los estudiantes en cuanto a su capacidad para identificar sus intereses vocacionales. Este factor quedó conformado por sólo tres items $(9,10$ y 11). Este último factor no agrupó items nuevos.

La consistencia interna del instrumento, así como de cada una de sus subescalas, fue estimada con el coeficiente Alfa de Cronbach. Los índices variaron de 0.79 (Autoconocimiento) a 0.88 (Planeación) en el caso de las subescalas. En tanto que para la puntuación total el índice fue de 0.95 (Tabla 1). 
Tabla 1. Indices de confiabilidad Alpha y número de items por subescala de la EAEC

\begin{tabular}{lll}
\hline Subescala & Items & Alpha \\
\hline Autoconocimiento & 11 & .79 \\
Información ocupacional & 10 & .86 \\
Establecimiento de metas & 13 & .87 \\
Solucion de problemas & 12 & .87 \\
Planeación & 9 & .88 \\
Escala total & 55 & .95
\end{tabular}

\section{Conclusiones}

La inclusión de tareas culturalmente apropiadas para los estudiantes de Bachillerato, redundó en una mejor reproducción de la estructura teórica de la autoeficacia en la elección de carrera. El enfoque teórico establece la existencia de cinco dimensiones que la definen. Dichas dimensiones fueron recreadas mediante la combinación de items originales e items nuevos. En el estudio reportado por Canto y Rodríguez (1998), la estructura teórica del constructo no fue reproducida y probablemente esto se debió principalmente a la inclusión de items que definían tareas que no resultaban significativas para los estudiantes de bachillerato de contextos hispanoamericanos. Por ejemplo, el item "Encontrar información en la biblioteca acerca de las ocupaciones que te interesan", no era culturalmente adecuada para la muestra, ya que las bibliotecas locales, a diferencia de las norteamericanas, carecen de ese tipo de información.

¿Cómo operó la falta de significancia en el intento fallido de recrear la estructura factorial de la autoeficacia en la elección de carrera?. Nosotros pensamos que una conducta típica cuando se desconoce el contexto de la pregunta es la sobrevaloración de la respuesta. Esta afirmación, ( $\sin$ apoyo teórico o empírico que la sustente) es una mera suposición de nuestra parte, aunque pensamos que es altamente plausible. Al utilizar preguntas que requerían de tareas no típicas, las respuestas dadas contenían una combinación de información acerca de la autoeficacia e información acerca del proceso individual de sobrevaloración. En la medida que la tarea se apartara aun más de las situaciones típicas de los estudiantes, sus respuestas pasarían a estar más explicadas por sus procesos individuales de sobrevaloración que por el atributo mismo de la autoeficacia. 
El proceso de sobrevaloración que se activa ante situaciones atípicas contribuye a la generación de dos posibles situaciones que afectan tanto al número de dimensiones reproducidas como el significado de las dimensiones. La primera situación está dada por la restricción de la escala del instrumento de medición. La conducta de sobrevaloración implica que los estudiantes en vez de seleccionar sus respuestas entre el valor mínimo de la escala (0) y el máximo (9), las pasaron a seleccionar posiblemente entre 6 y 9. La reducción en el rango de la escala afecta la magnitud de las correlaciones entre las variables y, por consiguiente, limita la reproducción de estructuras factoriales complejas.

La segunda situación está dada por la incorporación de error en la medición. Al sobreestimar, el estudiante está introduciendo error aleatorio que si bien crea variabilidad entre las puntuaciones observadas, está no puede ser atribuida a la diferencia en los niveles de autoeficacia en la elección de carrera de la muestra de estudiantes. La variabilidad observada pudo haber sido el resultado más bien de la variabilidad en los procesos de sobrevaloración de los estudiantes. Por consiguiente, el significado de las dimensiones estaría confundido con dicho proceso.

Por otra parte, en este estudio se pudo observar que la inclusión de tareas culturalmente apropiadas y típicas, posiblemente inhibió la conducta de sobrevaloración en las respuestas de los estudiantes, por lo que podríamos pensar que es una medida que podría ser explicada por la autoeficia. Sus efectos se hicieron visibles en la magnitud de las correlaciones alcanzadas y la reproducción de la estructura factorial de la autoeficacia en la elección de carrera.

La inclusión de tareas culturalmente apropiadas también influenció positivamente la fiabilidad de la información. La consistencia de las puntuaciones de la escala final para medir la autoeficacia en la elección de carrera resultó similar a la reportada en otros contextos culturales (Luzzo, 1993). Más aún, cuando se compararon con los resultados obtenidos con la versión corta traducida al español, los valores estimados de la consistencia interna de las cinco subescalas y la escala total resultaron significativamente mayores.

Sin embargo, habrá que reconocer que todavía queda una gran parte de la varianza sin explicar, ya que con este instrumento sólo se explicó poco más del $42 \%$ de la varianza; además, pudo observarse que fue el primer factor (Autoconocimiento) el que tuvo un mayor peso 
en la explicación de la autoeficacia para la elección de carrera. A partir de estos resultados, se sugiere realizar estudios dirigidos a observar con más detenimiento las características de los estudiantes que responden el cuestionario, ya que por ser un autoinforme, queda por observar sí efectivamente los que dicen que se consideran autoeficaces para elegir carrera, en realidad realizan las actividades que se supone les ayudaría a elegir su carrera adecuadamente. 


\section{Referencias}

Anderson, S. y Brown, C. (1997). Self-efficacy as a determinant of career maturity in urban and rural high school students. Journal of Career Assessment, 5 (3), 305-315.

Bandura, A. (1977). Self-efficacy: Toward a unifying theory of behavioral change. Psychological Review, 84 (1), 191-215.

.Bandura, A. (1986). Social foundations of thought and action. Englewood Cliff, NJ: Prentice Hall.

Bandura, A. (1997). Self-efficacy. The exercise of control. New York: Freeman and Company.

Betz, N. y Hackett, G. (1981). The relationship of career-related self-efficacy expectations to perceived career options in college women and men. Journal of Counseling Psychology, 28 (4), 399-410.

Betz, N. E., Klein, K. L., \& Taylor, K. M. (1996). Evaluation of a short form of the Career Decision-Making Self-Efficacy Scale. Journal of Career Assessment, 4 (1), 47-57.

Blustein, D. L. (1989). The concept of agency in educational and career development. Journal of Counseling Psychology, 35 (2), 194-203.

Bollen, K. (1989). Structural equations with latent variables. USA: Wiley Interscience.

Canto y Rodríguez J. (1998). Factors influencing certainty of career choice among preparatoria students at the Autonomous University of Yucatan. Tesis doctoral no publicada. Facultad de Psicología, Universidad Autónoma de Yucatán, Mérida, Yuc., México.

Carns, A., Carns, M., Wooten, H., Jones, L., Raffield, P., y Heitkamp, J. (1995). Extracurricular activities: are they beneficial? Texas Counseling Association Journal, 23 (2), 37-45 (1995).

Crites, J.O. (1978). Psicología vocacional. Argentina: Paidós.

Hackett, G. y Betz, N. (1981). A self-efficacy approach to the career development of women. Journal of Vocational Behavior, 18 (3), 326-339.

Hackett G. y Betz, N. (1995). Self-efficacy and career choice and development. En J. E. Maddux. (Ed.), Self-efficacy, adaptation and adjustment. Theory, research and application. (pp. 249-279). New York: Plenum Press.

Luzzo, D. A. (1993). Reliability and validity testing of the Career Decision-making SelfEfficacy Scale. Measurement and Evaluation in Counseling and Development, 26 (2), 137-142.

Luzzo, D. A. (1996). A psychometric evaluation of the Career Decision-making Self-Efficacy Scale. Journal of Counseling and Development, 74 (3), 276-279. 
McIntosh, P. I. (2000). Life Career Development: Implications for School Counselors. Journal of Education, 120 (4), 621-626.

Maddux, J. E. (Ed). (1995). Self-efficacy, adaptation and adjustment. Theory, research and application. New York: Plenum Press.

Pajares, F. (1996). Self-efficacy beliefs in academic settings. Review of Educational Research, 66 (4), 543-578.

Robbins, S. (1985). Validity estimates for the Career Decision-making Self-Efficacy Scale. Measurement and Evaluation in Counseling and Development, 18 (2), 64-71.

Taylor, K. y Betz, N. (1983). Applications of self-efficacy theory to the understanding and treatment of career indecision. Journal of Vocational Behavior, 22 (1), 63-81.

Taylor, K. y Popma, J. (1990). Construct validity of the Career Decision-Making SelfEfficacy Scale and the relationship of CDMSE to vocational indecision. Journal of Vocational Behavior, 37 (1), 17-31. 


\section{ANEXO 1 \\ ESCALA DE AUTOEFICACIA EN LA ELECCIÓN DE CARRERA}

Ésta es una escala que pretende identificar las creencias que tienen los estudiantes de preparatoria con respecto a su capacidad para realizar algunas tareas relacionadas con la elección de su carrera. Es muy importante que respondas de acuerdo a cuánta confianza tienes que podrías realizar la tarea que se te presenta. Los resultados de la aplicación de esta escala serán manejados en forma estrictamente confidencial. Aquí no hay respuestas correctas o incorrectas, por lo que puedes responder con toda honestidad. Lo que interesa son los resultados grupales y no los individuales.

INSTRUCCIONES: Lee cuidadosamente cada una de las siguientes afirmaciones y encierra en un círculo $\mathrm{O}$ en el lugar correspondiente de la hoja de respuestas, cuanta confianza tienes de que podrías realizar cada una de las tareas que se te presentan. Utiliza la escala del 0 al 9 que se te muestra enseguida.

$$
0
$$

Ninguna

confianza

$$
123
$$

Muy poca confianza
45

Algo de confianza $\begin{array}{ll}6 & 7\end{array}$

Mucha confianza
89

Confianza

absoluta

¿Cuánta confianza tienes de que podrías......

1. Evaluar con exactitud tus habilidades?

2. Determinar cuál sería tu trabajo ideal?

3. Determinar cuáles son tus valores?

4. Definir el estilo de vida que te gustaría tener?

5. Estimar lo que quieres ganar al desempeñar tu profesión?

6. Identificar las actividades que menos te gustan?

7. Identificar algunas características de tu personalidad?

8. Aceptar dejar algo importante para ti, a fin de alcanzar tus metas profesionales?

9. Identificar algunas carreras que te interesan?

10. Identificar algunas ocupaciones que te interesan?

11. Determinar si tienes preferencia por el trabajo con la gente o con información?

12. Encontrar información acerca de las ocupaciones que te interesan?

13. Hablar con una persona que este trabajando en la ocupación que te interesa? 
14. Obtener información acerca de escuelas profesionales o universitarias?

15. Asistir a alguna escuela a obtener información de las carreras que ofrecen?

16. Obtener información impresa de alguna de las Universidades del estado o del país?

17. Asistir a pláticas que proporcionen información acerca de las carreras que te interesan?

18. Revisar libros relacionados con las carreras que te interesan?

19. Asistir a algún evento (muestra, expo, etc.) vocacional?

20. Describir las características de las carreras que te interesan?

21. Preguntarle a algún maestro acerca de las oportunidades de trabajo?

22. Seleccionar una carrera entre varias en las que estás interesado?

23. Escoger una carrera que se adapte al estilo de vida de tu preferencia?

24. Seleccionar una ocupación de una lista que te interese?

25. Escoger una licenciatura que vaya de acuerdo con tus intereses?

26. Persistir en lo que te has propuesto hasta alcanzar tus metas profesionales?

27. Determinar el nivel académico que te gustaría obtener?

28. Encontrar la manera de obtener lo que quieres aunque tus circunstancias te sean desfavorables?

29. Definir lo estarás haciendo dentro de cinco años?

30. Escoger una licenciatura que tus padres no aprueban?

31. Elegir una carrera en la que la mayoría de los trabajadores son del sexo opuesto?

32. Elegir una carrera que este de acuerdo con tus habilidades?

33. Elegir la mejor carrera para ti, a pesar de que te lleve más tiempo terminarla?

34. Describir el ambiente laboral en el que te gustaría trabajar?

35. Determinar los pasos a seguir si tuvieras problemas en la licenciatura que elegiste?

36. Cambiar de licenciatura si NO te gusta la primera que elegiste?

37. Cambiar de empleo si no estás satisfecho?

38. Encontrar alternativas de carrera si no pudieras ingresar a la que elegiste primero? 
39. Encontrar alternativas de solución si te encontraras en una situación difícil?

40. Resolver algún problema académico?

41.Permanecer tranquilo cuando te encuentras en dificultades en la escuela?.

42. Manejar adecuadamente acontecimientos inesperados?

43. Regresar a la escuela después de algunos años de haberla abandonado?

44. Tener una estrategia en caso de que te dieran de baja de la escuela?

45. Solicitar ingreso a la Universidad después de haber sido rechazado la primera vez?

46. Resistir las presiones de tus padres y amigos para elegir una carrera que no te gusta?

47. Hacer un plan de tus metas vocacionales para los próximos cinco años?

48. Determinar los pasos que necesitas realizar para terminar con éxito la licenciatura que elijas?

49. Planear sus actividades del semestre, del mes o del día?

50. Utilizar una agenda o libreta para no olvidar tus compromisos escolares?

51. Establecer una fecha para realizar algún plan personal?

52. Tomar cursos extracurriculares que te servirán en tu carrera?

53. Relacionarte con personas ligadas a la carrera que deseas estudiar?

54. Jerarquizar tus actividades en orden de importancia de acuerdo a tus planes académicos?

55. Hablar con alguien de tus planes vocacionales futuros? 


\section{ANEXO 2}

Matriz factorial de la Escala de Autoeficacia en la Elección de Carreral (Establecimiento de metas), factores, carga factorial y comunalidades.

\begin{tabular}{|c|c|c|c|}
\hline Ítem & Factor 1. Establecimiento de metas & $\begin{array}{c}\text { Carga } \\
\text { factorial }\end{array}$ & $\begin{array}{c}\text { Comunalid } \\
\text { ades }\end{array}$ \\
\hline 2 & Determinar cual sería tu trabajo ideal. & .5872 & .769 \\
\hline 4 & Definir el estilo de vida que te gustaría tener. & .4161 & .495 \\
\hline 22 & Seleccionar una carrera entre varias en las que estas interesado. & .6628 & .742 \\
\hline 23 & $\begin{array}{l}\text { Escoger una carrera que se adapte al estilo de vida de tu preferen- } \\
\text { cia. }\end{array}$ & .6540 & .723 \\
\hline 24 & Seleccionar una ocupación de una lista que te interese. & .6149 & .764 \\
\hline 25 & Escoger una licenciatura que vaya de acuerdo con tus intereses. & .6360 & .769 \\
\hline 26 & $\begin{array}{l}\text { Persistir en lo que te has propuesto hasta alcanzar tus metas profe- } \\
\text { sionales. }\end{array}$ & .5223 & .712 \\
\hline 27 & Determinar el nivel académico que te gustaría obtener. & .5352 & .685 \\
\hline 28 & $\begin{array}{l}\text { Encontrar la manera de obtener lo que quieres aunque tus circuns- } \\
\text { tancias no te sean favorables. }\end{array}$ & .5038 & .677 \\
\hline 29 & Definir lo que estarás haciendo dentro de cinco años. & .4479 & .599 \\
\hline \multirow[t]{2}{*}{34} & Describir el ambiente laboral en el que te gustaría trabajar. & .4157 & .727 \\
\hline & Factor 2. Solución de problemas & $\begin{array}{c}\text { Carga } \\
\text { factorial }\end{array}$ & $\begin{array}{l}\text { Comunali- } \\
\text { des }\end{array}$ \\
\hline 35 & $\begin{array}{l}\text { Determinar los pasos a seguir si estuvieras teniendo problemas en } \\
\text { la licenciatura que elegiste. }\end{array}$ & .5769 & .711 \\
\hline 36 & Cambiar de licenciatura si no te gusta la primera que elegiste. & .4723 & .678 \\
\hline 37 & Cambiar de empleo si no estas satisfecho. & .4538 & .646 \\
\hline 38 & $\begin{array}{l}\text { Encontrar alternativas de carrera si no pudieras ingresar a la que } \\
\text { elegiste primero. }\end{array}$ & .5483 & .635 \\
\hline 39 & $\begin{array}{l}\text { Encontrar alternativas de solución si te encontraras en una situación } \\
\text { difícil. }\end{array}$ & .5953 & .637 \\
\hline 40 & Resolver algún problema académico. & .6933 & .668 \\
\hline 41 & $\begin{array}{l}\text { Permanecer tranquilo cuando te encuentras en dificultades en la } \\
\text { escuela. }\end{array}$ & .6185 & .659 \\
\hline 42 & Manejar adecuadamente acontecimientos inesperados. & .5773 & .737 \\
\hline 43 & $\begin{array}{l}\text { Regresar a la escuela después de algunos años de haberla abando- } \\
\text { nado. }\end{array}$ & .5910 & .789 \\
\hline 44 & Tener una estrategia en caso de que te dieran de baja en la escuela. & .6323 & .539 \\
\hline 45 & $\begin{array}{l}\text { Solicitar ingreso a la Universidad después de haber sido rechazado } \\
\text { la primera vez. }\end{array}$ & .4569 & .685 \\
\hline
\end{tabular}




\begin{tabular}{|c|c|c|c|}
\hline & Factor 3. Información ocupacional & $\begin{array}{c}\text { Carga } \\
\text { factorial }\end{array}$ & $\begin{array}{c}\text { Comunalid } \\
\text { ades }\end{array}$ \\
\hline 12 & Encontrar información acerca de las ocupaciones que te interesan. & .4625 & .658 \\
\hline 13 & $\begin{array}{l}\text { Hablar con una persona que este trabajando en la ocupación que te } \\
\text { interesa. }\end{array}$ & .5471 & .644 \\
\hline 14 & $\begin{array}{l}\text { Obtener información acerca de escuelas profesionales o universita- } \\
\text { rias. }\end{array}$ & .4019 & .647 \\
\hline 15 & $\begin{array}{l}\text { Asistir a alguna escuela a obtener información de las carreras que } \\
\text { ofrecen. }\end{array}$ & .7348 & .618 \\
\hline 16 & $\begin{array}{l}\text { Obtener información impresa de alguna de las universidades del } \\
\text { estado o del país. }\end{array}$ & .5807 & .659 \\
\hline 17 & $\begin{array}{l}\text { Asistir a pláticas que proporcionen información acerca de las carre- } \\
\text { ras que te interesan. }\end{array}$ & .7093 & .716 \\
\hline 18 & Revisar libros relacionados con las carreras que te interesan. & .4903 & 690 \\
\hline 19 & Asistir a algún evento (muestra, expo, etc.,) vocacional. & .5414 & .673 \\
\hline \multirow[t]{2}{*}{21} & Preguntarle a algún maestro acerca de las oportunidades de trabajo. & .4393 & .682 \\
\hline & Factor 4. Planeación & $\begin{array}{c}\text { Carga } \\
\text { factorial }\end{array}$ & $\begin{array}{c}\text { Comunalid } \\
\text { ades }\end{array}$ \\
\hline 47 & $\begin{array}{l}\text { Hacer un plan de tus metas vocacionales para los próximos cinco } \\
\text { años. }\end{array}$ & .4841 & .598 \\
\hline 48 & $\begin{array}{l}\text { Determinar los pasos que necesitas realizar para terminar con éxito } \\
\text { la licenciatura que elijas. }\end{array}$ & .4936 & .605 \\
\hline 49 & Planear tus actividades del semestre, mes o día. & .5749 & .643 \\
\hline 50 & $\begin{array}{l}\text { Utilizar una agenda o libreta para no olvidar tus compromisos esco- } \\
\text { lares. }\end{array}$ & .6083 & .636 \\
\hline 51 & Establecer una fecha para realizar algún plan personal. & .6533 & .631 \\
\hline 52 & Tomar cursos extracurriculares que te servirán en tu carrera. & .5326 & .699 \\
\hline 53 & Relacionarte con personas ligadas a la carrera que deseas estudiar. & .5331 & .661 \\
\hline 54 & $\begin{array}{l}\text { Jerarquizar tus actividades en orden de importancia de acuerdo a } \\
\text { tus planes académicos. }\end{array}$ & .5399 & .717 \\
\hline \multirow[t]{2}{*}{55} & Hablar con alguien de tus planes vocacionales futuros & .4235 & .732 \\
\hline & Factor 5. Autoconocimiento & $\begin{array}{c}\text { Carga } \\
\text { factorial }\end{array}$ & $\begin{array}{c}\text { Comunali- } \\
\text { dades }\end{array}$ \\
\hline 9 & Identificar algunas carreras que te interesan. & .6440 & .583 \\
\hline 10 & Identificar algunas ocupaciones que te interesan. & .7903 & .650 \\
\hline 11 & $\begin{array}{l}\text { Determinar si tienes preferencia por el trabajo con la gente o con la } \\
\text { información. }\end{array}$ & .5311 & .532 \\
\hline
\end{tabular}

